\title{
Prior use of therapeutic anticoagulation does not protect against COVID-19 related clinical outcomes in hospitalized patients: a propensity score-matched cohort study
}

\author{
Janneke Spiegelenberg ${ }^{1}$, Marleen van Gelder ${ }^{2}$, Martje Maas $^{3}$, marcel Hovens ${ }^{4}$, Anne \\ Esselink $^{5}$, Anton Dofferhoff ${ }^{6}$, Rob Janssen ${ }^{6}$, Josephine van de Maat ${ }^{1}$, Jacobien \\ Hoogerwerf $^{1}$, Nico Janssen ${ }^{2}$, Marianne van Apeldoorn ${ }^{7}$, Angèle Kerckhoffs ${ }^{8}$, Karin \\ Veerman $^{9}$, Marc Blaauw ${ }^{1}$, Robert Jan Hassing ${ }^{4}$, Kees Kramers ${ }^{10}$, and Jenneke Leentjens ${ }^{1}$ \\ ${ }^{1}$ Affiliation not available \\ ${ }^{2}$ Radboudumc \\ ${ }^{3}$ Bernhoven Hospital Location Uden \\ ${ }^{4}$ Hospital Rijnstate Arnhem \\ ${ }^{5}$ Canisius Wilhelmina Hospital \\ ${ }^{6}$ Canisius Wilhelmina Ziekenhuis \\ ${ }^{7}$ Jeroen Bosch Hospital \\ ${ }^{8}$ Jeroen Bosch Ziekenhuis \\ ${ }^{9}$ Sint Maartenskliniek \\ ${ }^{10}$ Radboud Universiteit
}

January 19, 2021

\begin{abstract}
The hypercoagulable state observed in COVID-19 could be responsible for morbidity and mortality. In this retrospective study we investigated whether therapeutic anticoagulation prior to infection has a beneficial effect in hospitalized COVID-19 patients. 1154 COVID-19 patients admitted to 6 hospitals in the Netherlands between March and May 2020 were included. We applied 1:3 propensity score matching to evaluate the association between prior therapeutic anticoagulation use and clinical outcome, with in hospital mortality as primary endpoint. 190 (16\%) patients used therapeutic anticoagulation prior to admission. In the propensity score matched analyses, we observed no associations between prior use of therapeutic anticoagulation and overall mortality (RR 1.02 (95\% CI; 0.80-1.30) or length of hospital stay (7.0 [4-12] vs 7.0 \{4-12] days, p=0.69), although we observed a lower risk of pulmonary embolism (RR 0.19 (95\% CI; 0.05-0.80). This study shows that prior use of therapeutic anticoagulation is not associated with improved clinical outcome in hospitalized COVID-19 patients.
\end{abstract}

Prior use of therapeutic anticoagulation does not protect against COVID-19 related clinical outcomes in hospitalized patients: a propensity score-matched cohort study

J.P. Spiegelenberg ${ }^{1,2}$, M.M.H.J. van Gelder ${ }^{3,4}$, M.L. Maas ${ }^{5}$, M.M.C. Hovens ${ }^{6}$, A. Esselink ${ }^{7}$, A.S.M. Dofferhoff $^{7}$, R. Janssen ${ }^{8}$, J. van de Maat ${ }^{1,2}$, N. Janssen ${ }^{1,2}$, M. Blaauw ${ }^{5}$, R.J. Hassing ${ }^{6}$, M. van Apeldoorn ${ }^{9}$, A. Kerckhoffs ${ }^{9}$, K. Veerman ${ }^{10}$, J. Hoogerwerf ${ }^{1,2}$, C. Kramers ${ }^{11,12}$, J. Leentjens ${ }^{1,2}$

${ }^{1}$ Department of Internal Medicine, Radboud university medical center, 6500HB Nijmegen, the Netherlands

2 Radboud Center for Infectious Diseases, Radboud university medical center, 6500HB Nijmegen, The Netherlands 
3 Department for Health Evidence, Radboud Institute for Health Sciences, Radboud university medical center, 6500HB Nijmegen, the Netherlands

${ }^{4}$ Radboud REshape Innovation Center, Radboud university medical center, 6500HB Nijmegen, the Netherlands

${ }^{5}$ Department of Internal Medicine, Bernhoven, 5406PT Uden, the Netherlands

${ }^{6}$ Department of Internal Medicine, Rijnstate Hospital, 6815AD Arnhem, the Netherlands

7 Department of Internal Medicine, Canisius-Wilhelmina Hospital, 6500GS Nijmegen, The Netherlands

8 Department of Pulmonary Medicine, Canisius-Wilhelmina Hospital, 6500GS Nijmegen, The Netherlands

9 Department of Internal Medicin and Geriatricts, Jeroen Bosch Hospital, 5223 GZ, 's-Hertogenbosch, the Netherlands

10 St. Maartenskliniek Department of Internal Medicine, Hengstdal 36574 NA Ubbergen, The Netherlands

11 Department of Pharmacology-Toxicology, Radboud university medical center, 6500HB Nijmegen, the Netherlands

12 Department of Pharmacy, Canisius Willhelmina Hospital, 6500GS Nijmegen, the Netherlands

\section{Correspondence}

Jenneke Leentjens, MD, PhD

Radboud university medical center

E-mail: jenneke.leentjens@radboudumc.nl

\section{Conflicts of interest/Competing interests}

R Janssen discloses application of a patent on vitamin K in COVID-19. R Janssen and ASM Dofferhoff have a scientific collaboration with Kappa Bioscience AS, a manufacturer of vitamin K2 (MK-7).

\section{Funding}

The study was internally funded by the participating departments.

\section{Ethics approval}

The study was carried out in the Netherlands in accordance with the applicable rules concerning the review of research ethics committees and informed consent.

\section{Availability of data and material}

The datasets generated during and/or analyzed during the current study are available from the corresponding author on reasonable request.

\section{Authors' contributions}

JS, MG, MM, MH, AE, AD, RJ, CK, and JL designed the study. AD, JvdM, JH, NJ, MvA, AK, KV, MB, and RJH were responsible for sample collection and laboratory processing. JS, AD, RJ, JvdM, JH, NJ, MvA, AK, KV, MB, RJH, and JL were responsible for data collection and management. JS, MG, CK, and JL performed the statistical analyses and drafted the manuscript. MM, MH, AE, AD, RJ, JvdM, JH, NJ, MvA, AK, KV, MB, and RJH critically revised the manuscript. All authors read and approved the final manuscript.

Key words: Corona virus disease 2019, thrombosis, pulmonary embolism, thromboprophylaxis, anticoagulation, DOAC, Vitamin K antagonist.

Word count: 1964 


\begin{abstract}
The hypercoagulable state observed in COVID-19 could be responsible for morbidity and mortality. In this retrospective study we investigated whether therapeutic anticoagulation prior to infection has a beneficial effect in hospitalized COVID-19 patients. 1154 COVID-19 patients admitted to 6 hospitals in the Netherlands between March and May 2020 were included. We applied 1:3 propensity score matching to evaluate the association between prior therapeutic anticoagulation use and clinical outcome, with in hospital mortality as primary endpoint. $190(16 \%)$ patients used therapeutic anticoagulation prior to admission. In the propensity score matched analyses, we observed no associations between prior use of therapeutic anticoagulation and overall mortality (RR 1.02 (95\% CI; 0.80-1.30) or length of hospital stay (7.0 [4-12] vs 7.0 \{4-12] days, $\mathrm{p}=0.69$ ), although we observed a lower risk of pulmonary embolism (RR 0.19 (95\% CI; 0.05-0.80). This study shows that prior use of therapeutic anticoagulation is not associated with improved clinical outcome in hospitalized COVID-19 patients.
\end{abstract}

\title{
What is already known about this subject
}

We searched PubMed for articles published from the start of the pandemic up to October $30^{\text {th }}, 2020$, using the keywords "coronavirus", "COVID-19", "SARS-CoV-2", "therapeutic anticoagulation", and "thrombosis" or "embolism". The existing evidence shows that patients with COVID-19 have a hypercoagulable state with increased risk of thrombotic events. Three studies investigated the association between therapeutic anticoagulation prior to hospitalization and mortality with ambivalent results, probably due to methodological limitations.

\section{What this study adds}

A rigorous statistical analysis with thorough adjustment for confounding to properly investigate the treatment effect of prior therapeutic anticoagulation on different clinically relevant outcomes in a large cohort of hospitalized COVID-19 patients. Moreover, we are the first to show that prior use of therapeutic anticoagulation is not associated with better outcome parameters in hospitalized COVID-19 patients in terms of all-cause mortality, ICU admittance, need for mechanical ventilation, and length of hospital stay.

\section{Introduction}

Coronavirus disease 2019 (COVID-19) is an infectious disease caused by the severe acute respiratory syndrome coronavirus-2 (SARS-CoV-2). Besides respiratory failure, COVID-19 also has high rates of thromboembolic complications as seen in multiple retrospective studies. ${ }^{1-3}$ In accordance, autopsy studies showed high incidences of macro- and microemboli in patients who were infected by SARS-CoV-2. ${ }^{4,5}$ These thrombotic complications could be responsible for a significant part of the morbidity and mortality seen in COVID19 patients. ${ }^{3}$ The exact underlying pathophysiology of COVID-19 related thrombotic complications remains unknown, but excessive inflammation, hypoxia, immobilization, thrombotic microangiopathy, diffuse intravascular coagulation, and complement activation most likely play a role. ${ }^{6}$ Moreover, low-molecular-weight heparin (LMWH) thromboprophylaxis seems to decrease mortality in patients with COVID-19. ${ }^{15}$

Based on these data, guidelines recommend administration of prophylactic LMWH for all hospitalized patients with COVID-19. ${ }^{16,17}$ However, recent reports show that despite the use of apparently adequate thrombosis prophylaxis, the incidence of venous thromboembolism (VTE) in COVID-19 patients who were admitted to a hospital ward appears to be much higher compared to patients with other infectious diseases. ${ }^{2,18,19}$ Therefore, it was suggested that in COVID-19 patients increased intensity thromboprophylaxis or therapeutic anticoagulation should be considered. ${ }^{16,21,22}$ However, studies that address the effect of therapeutic anticoagulation on clinical outcomes show conflicting results, and often compare very heterogeneous cohorts that lack statistical power to draw firm conclusions. ${ }^{23-27}$ The aim of this study is to investigate the beneficial effect of therapeutic anticoagulation used prior to hospitalization on morbidity and mortality in a large cohort of hospitalized COVID-19 patients.

\section{Methods}




\section{Patients}

We included all patients [?]18 years old with confirmed COVID-19 admitted to one of the six participating hospitals in the Netherlands (1 academic hospital (Radboudumc, Nijmegen), 5 general hospitals (Bernhoven hospital, Uden; Sint Maartenskliniek, Nijmegen; Canisius Wilhelmina hospital, Nijmegen; Rijnstate hospital, Arnhem; Jeroen Bosch hospital, 's-Hertogenbosch)) between March 1 and May 31, 2020. The diagnosis COVID-19 was made with a real-time reverse transcriptase polymerase chain reaction (RT-PCR) positive for SARS-CoV-2 on a deep naso-oropharyngeal swab. In addition, patients with negative PCR but with clinical symptoms consistent with COVID-19 and a CT-scan of the chest showing a very high suspicion of typical pulmonary involvement of COVID-19 (COVID-19 reporting and data system score of 5 defined by the Dutch Radiology Society) were included. ${ }^{29}$ Patients were excluded when COVID-19 was not RT-PCR or radiographically confirmed, or when patients had insufficient clinical documentation because they were transferred to or from another hospital due to capacity constraints.

The index date was the day of hospital admission. Patients were followed until hospital discharge or death. Data on the occurrence of thrombotic events, length of hospital stay, ICU admission, type of oxygen ventilation, and mortality were obtained from the patients' records (EPIC, EPIC Systems Corporation, Verona, Wisconsin, USA; HiX, ChipSoft, Amsterdam, The Netherlands; xCare EPD, NEXUS, Nieuwegein, The Netherlands) and recorded in our database using a standardized case report form in the good clinical practice (GCP)-compliant data management system Castor (Castor Electronic Data Collection, Amsterdam, the Netherlands). CT pulmonary angiograms (CTPAs) and Doppler ultrasonography were performed at the discretion of the treating clinician. Common indications for CTPA included high D-dimer levels and/or progressive hypoxemia. The study was carried out in the Netherlands in accordance with the applicable rules concerning the review of research ethics committees and informed consent. The Institutional Review Boards of the participating hospitals waived the need for informed consent due to the observational nature of this study.

\section{Outcomes}

The primary outcome was all-cause in hospital mortality. Secondary outcomes included admission to the ICU, need for invasive mechanical ventilation, critical respiratory status (defined as a composite endpoint of the need for invasive mechanical ventilation and/or need of venturi mask and/or non-rebreathing mask), imaging proved pulmonary embolism (PE), and length of hospital stay.

\section{Potential confounders}

Potential confounders were identified literature review. Directed acyclic graphs (DAG) were subsequently drawn to visualize causal assumptions to identify confounders (Figure S1 in the online supplement). Age, sex, body mass index (BMI), medical history of chronic pulmonary disease, diabetes mellitus, active malignancy, hypertension, obstructive coronary heart disease, myocardial infarction, nonischaemic cardiomyopathy, heart failure, previous heart surgery, electronic heart device, cerebrovascular accidents, and/or peripheral artery disease, use of immunosuppressive medication, and no-ICU policy were identified as confounders that were available in our database.

\section{Statistical analysis}

Descriptive statistics were used to compare the patients with and without prior therapeutic anticoagulation and to estimate the prevalence of the outcomes. Categorical parameters were presented as counts with percentages, continuous parameters with medians and interquartile ranges, based on their non-normal distribution tested with the Shapiro-Wilk test. Comparisons were performed using Mann-Whitney U test or Chi-Squared test as appropriate. In order to compare the outcomes while adjusted for potential confounding resulting from the non-randomized design of our observational study, we applied propensity score-matching methods. In our database, 174 (15\%) patients had missing information for BMI. Therefore, we first imputed BMI values with single imputation using predicted values from multivariable models including age, sex, hypertension, diabetes mellitus, and the outcome mortality. Propensity scores were generated using 
a multivariable logistic regression model with prior therapeutic anticoagulation use as outcome and the 17 variables previously stated as predictors. The patients with therapeutic anticoagulation were subsequently matched in a 1:3 ratio with patients without therapeutic anticoagulation prior to admission on these propensity scores with Nearest Neighbor Matching techniques without replacement and a caliper width of $0 \cdot 1$ of the standard deviation of the logit of the propensity score. To evaluate the balance of measured confounders between exposed and unexposed groups, we calculated the standardized mean difference (SMD). An SMD $<0.25$ indicated balance of matched cohorts. ${ }^{30}$

Associations between prior therapeutic use of anticoagulants and the outcomes overall in hospital mortality, admission to ICU, occurrence of PE, critical respiratory state, and the need for invasive mechanical ventilation were estimated as risk ratios (RR) with $95 \%$ confidence intervals (CI). Estimating the differences in length of hospital stay between the two groups was performed using Mann-Whitney U test stratified by mortality.

In our secondary analysis, we compared VKA versus no therapeutic anticoagulation, DOAC versus no therapeutic anticoagulation and VKA versus DOAC on the before mentioned outcome parameters. Matching was performed using R-software/studio Version 1.3.1093 and statistical analysis were performed using STATA/SE 16.0 (Stata Corp, TX, USA).

\section{Results}

Of the 1316 patients who were hospitalized with proven COVID-19 between 1th of March and $31^{\text {st }}$ of May 2020, 239 patients were excluded because of reasons depicted in Figure 1. Of the 1154 patients included in this study, $92(8 \%)$ used VKA and $98(8 \%)$ used DOAC, and $964(84 \%)$ patients did not use therapeutic anticoagulation prior to COVID-19 diagnosis. All patients in the exposed group were continued on therapeutic anticoagulation during hospitalization. Among patients who did not use therapeutic anticoagulation prior to admission, 856 (89\%) received prophylactic LMWH during hospitalization. COVID-19 was confirmed by a positive PCR test in 1124 (97\%) patients or considered confirmed by clinical features in combination with a CT scan with a very high level of suspicion (CO-RADS 5) in $30(3 \%)$ patients.

Baseline patient characteristics are shown in table 1. Patients who used therapeutic anticoagulation prior to hospitalization were older, more likely of male sex, and more likely to have cardiovascular comorbidities or a no-ICU policy compared to patients who did not use prior therapeutic anticoagulation. Subsequent propensity score matching retained $164(86 \%)$ patients who used prior therapeutic anticoagulation and 410 unexposed patients. The main covariates were balanced between the groups after the propensity score matching (table 1).

The results from the total cohort and the propensity score-matched analysis on the associations between therapeutic anticoagulation use and the dichotomous outcomes are presented in table $2 \mathrm{~A}$. In the crude total cohort analysis, therapeutic anticoagulation use was associated with an increased risk of mortality and decreased risks of ICU admission, mechanical ventilation, and PE. In the propensity score-matched analyses, however, no associations between therapeutic anticoagulation use and the outcomes were observed, except for a decreased risk of PE among patient with therapeutic anticoagulation (RR 0[?]19 (95\% CI 0[?]05-0[?]80)) (Table 2, supplementary Figure S2). In addition, therapeutic anticoagulation use was not associated with length of hospital stay (table 2B).

Similar to the primary analysis, no associations were found between VKA or DOAC use prior to admission and COVID-19 related clinical outcome parameters after propensity score matching (see tables S1-S6, in the online supplement).

\section{Discussion}

The main findings of this study are that therapeutic anticoagulation used prior to SARS-CoV-2 infection lowers the risk of pulmonary embolism but does not have an effect on other COVID-19 related outcomes in hospitalized COVID-19 patients, including in-hospital mortality. In addition, we did not observe differences in outcomes between DOAC or VKA-treated subgroups. 
The acute inflammatory phenomenon in COVID-19 amplifies hypercoagulability and increases the risk of thrombosis even under prophylaxis of LMWH. ${ }^{21}$ It has been hypothesized that therapeutic anticoagulation used prior to infection could improve the prognosis of COVID-19 by hampering coagulation activation. Indeed, previous studies showed that the use of therapeutic anticoagulation at hospital admission resulted in a lower incidence of VTE compared to thromboprophylaxis alone. ${ }^{23,24}$ Other studies, however, showed ambivalent results on COVID-19 severity and mortality due to comparison of dissimilar cohorts and lack of proper statistical adjustments for imbalances in baseline characteristics including comorbidities. ${ }^{26,27,31}$ Tremblay et al. also used a propensity score-matched comparison and found no statistically significant difference in mortality, time to mechanical ventilation, or hospitalization when comparing patients with and without therapeutic oral anticoagulation prior to SARS-CoV-2 infection. ${ }^{27}$ However, they included both ambulatory and hospitalized patients, and only adjusted for age, sex, race, Charlson Comorbidity Index and obesity in their propensity-score analysis while we illustrated that adjustment for more potential confounders is relevant. Moreover, they did not include thrombotic complications as an outcome parameter whereas our study showed a benefit on PE incidence, but not on other clinical endpoints.

This is also the first study that investigated the effects of therapeutic anticoagulation subgroups, i.e. vitamin $\mathrm{K}$ antagonists (VKAs) and direct oral anticoagulants (DOACs), in hospitalized COVID-19 patients. There are different hypotheses why VKAs could have unfavorable effects, and DOACs on the other hand could be of benefit in COVID-19. Dofferhoff et al detected reduced extrahepatic vitamin K status in patients with COVID-19 and showed that low vitamin K status was related to poor prognosis in these patients. ${ }^{28}$ VKA's are evident causes of reduced vitamin K status, but the relationship between VKA versus other therapeutic anticoagulation on the prognosis of COVID-19 patients has never been studied before.

Our study has several limitations that need to be addressed. Most importantly, its observational and retrospective nature limit causal inference, although the propensity score matching increases the credibility of our observations. Noteworthy, propensity score matching is often criticized because of its dependence on the included covariates. Confounders not included in the propensity score could lead to significant bias. However, in our study the prior visualization of relevant covariates in the DAG and the subsequent high and relevant number of included covariates in the propensity score matching, make this a valid approach. Other limitations are that there was no routine screening for pulmonary embolism, which may have resulted in underdiagnosis of this outcome especially in patients already treated with therapeutic anticoagulation.

Strengths of our study include the rigorous statistical analysis with thorough adjustment for confounding to properly investigate the treatment effect of prior therapeutic anticoagulation on different clinically relevant outcomes in a large cohort of hospitalized COVID-19 patients. Furthermore, we are the first to investigate the effect of therapeutic anticoagulation subgroups, i.e. vitamin K antagonists and DOACs.

In summary, although prior therapeutic anticoagulation use is associated with reduced PE occurrence, it is not associated with better outcome parameters in hospitalized COVID-19 patients in terms of all-cause mortality, ICU admittance, need for mechanical ventilation, and length of hospital stay. Secondary analysis between subgroups also showed no differences in clinical outcomes between VKA or DOAC treated patients.

\section{References}

1. Poissy J, Goutay J, Caplan M, et al. Pulmonary Embolism in Patients With COVID-19: Awareness of an Increased Prevalence. Circulation . 2020;142 (2):184-6.

2. Lodigiani C, Iapichino G, Carenzo L, et al. Venous and arterial thromboembolic complications in COVID19 patients admitted to an academic hospital in Milan, Italy. Thromb Res . 2020;191:9-14.

3. Klok FA, Kruip M, van der Meer NJM, et al. Incidence of thrombotic complications in critically ill ICU patients with COVID-19. Thromb Res . 2020;191:145-7.

4. Wichmann D, Sperhake JP, Lutgehetmann M, et al. Autopsy Findings and Venous Thromboembolism in Patients With COVID-19: A Prospective Cohort Study. Ann Intern Med . 2020;173 (4):268-77. 
5. Dolhnikoff M, Duarte-Neto AN, de Almeida Monteiro RA, et al. Pathological evidence of pulmonary thrombotic phenomena in severe COVID-19. J Thromb Haemost . 2020;18 (6):1517-9.

6. Belen-Apak FB, Sarialioglu F. Pulmonary intravascular coagulation in COVID-19: possible pathogenesis and recommendations on anticoagulant/thrombolytic therapy. J Thromb Thrombolysis.2020;50 (2):278-80.

7. Chen N, Zhou M, Dong X, et al. Epidemiological and clinical characteristics of 99 cases of 2019 novel coronavirus pneumonia in Wuhan, China: a descriptive study. Lancet.2020;395 (10223):507-13.

8. Guan WJ, Ni ZY, Hu Y, et al. Clinical Characteristics of Coronavirus Disease 2019 in China. $N$ Engl $J$ Med . 2020;382 (18):1708-20.

9. Huang C, Wang Y, Li X, et al. Clinical features of patients infected with 2019 novel coronavirus in Wuhan, China. Lancet . 2020;395 (10223):497-506.

10. Wang D, Hu B, Hu C, et al. Clinical Characteristics of 138 Hospitalized Patients With 2019 Novel Coronavirus-Infected Pneumonia in Wuhan, China. JAMA . 2020;323 (11):1061-9.

11. Sakka M, Connors JM, Hekimian G, et al. Association between D-Dimer levels and mortality in patients with coronavirus disease 2019 (COVID-19): a systematic review and pooled analysis. J Med Vasc . 2020;45 (5):268-74.

12. Zhang L, Yan X, Fan Q, et al. D-dimer levels on admission to predict in-hospital mortality in patients with Covid-19. J Thromb Haemost . 2020;18 (6):1324-9.

13. Tang N, Li D, Wang X, Sun Z. Abnormal coagulation parameters are associated with poor prognosis in patients with novel coronavirus pneumonia. J Thromb Haemost . 2020;18 (4):844-7.

14. Zhou F, Yu T, Du R, et al. Clinical course and risk factors for mortality of adult inpatients with COVID-19 in Wuhan, China: a retrospective cohort study. Lancet.2020;395 (10229):1054-62.

15. Thachil J. The versatile heparin in COVID-19. J Thromb Haemost . 2020;18 (5):1020-2.

16. Moores LK, Tritschler T, Brosnahan S, et al. Prevention, Diagnosis, and Treatment of VTE in Patients With Coronavirus Disease 2019: CHEST Guideline and Expert Panel Report. Chest . 2020;158 (3):1143-63.

17. Spyropoulos AC, Levy JH, Ageno W, et al. Scientific and Standardization Committee communication: Clinical guidance on the diagnosis, prevention, and treatment of venous thromboembolism in hospitalized patients with COVID-19. J Thromb Haemost.2020;18 (8):1859-65.

18. Artifoni M, Danic G, Gautier G, et al. Systematic assessment of venous thromboembolism in COVID19 patients receiving thromboprophylaxis: incidence and role of $\mathrm{D}$-dimer as predictive factors. $J$ Thromb Thrombolysis . 2020;50 (1):211-6.

19. Middeldorp S, Coppens M, van Haaps TF, et al. Incidence of venous thromboembolism in hospitalized patients with COVID-19. J Thromb Haemost . 2020;18 (8):1995-2002.

20. Helms J, Tacquard C, Severac F, et al. High risk of thrombosis in patients with severe SARS-CoV-2 infection: a multicenter prospective cohort study. Intensive Care Med . 2020;46 (6):1089-98.

21. Klok FA, Kruip M, van der Meer NJM, et al. Confirmation of the high cumulative incidence of thrombotic complications in critically ill ICU patients with COVID-19: An updated analysis. Thromb Res . 2020;191 :148-50.

22. Susen S, Tacquard CA, Godon A, et al. Prevention of thrombotic risk in hospitalized patients with COVID-19 and hemostasis monitoring. Crit Care . 2020;24 (1):364.

23. Lachant DJ, Lachant NA, Kouides P, Rappaport S, Prasad P, White RJ. Chronic therapeutic anticoagulation is associated with decreased thrombotic complications in SARS-CoV-2 infection. Journal of thrombosis and haemostasis . 2020;18 (10):2640-5. 
24. Llitjos JF, Leclerc M, Chochois C, et al. High incidence of venous thromboembolic events in anticoagulated severe COVID-19 patients.J Thromb Haemost . 2020;18 (7):1743-6.

25. Paranjpe I, Fuster V, Lala A, et al. Association of Treatment Dose Anticoagulation With In-Hospital Survival Among Hospitalized Patients With COVID-19. J Am Coll Cardiol . 2020;76 (1):122-4.

26. Rivera-Caravaca JM, Nunez-Gil IJ, Vivas D, et al. Clinical profile and prognosis in patients on oral anticoagulation before admission for COVID-19. Eur J Clin Invest . 2020:e13436.

27. Tremblay D, van Gerwen M, Alsen M, et al. Impact of anticoagulation prior to COVID-19 infection: a propensity score-matched cohort study.Blood . 2020;136 (1):144-7.

28. Dofferhoff ASM, Piscaer I, Schurgers LJ, et al. Reduced vitamin K status as a potentially modifiable risk factor of severe COVID-19. Clin Infect Dis. 2020.

29. De Smet K, De Smet D, Ryckaert T, et al. Diagnostic Performance of Chest CT for SARS-CoV-2 Infection in Individuals with or without COVID-19 Symptoms. Radiology. 2020:202708.

30. Stuart EA, Lee BK, Leacy FP. Prognostic score-based balance measures can be a useful diagnostic for propensity score methods in comparative effectiveness research. J Clin Epidemiol . 2013;66 (8 Suppl):S84S90 e1.

31. Schiavone M, Gasperetti A, Mancone M, et al. Oral anticoagulation and clinical outcomes in COVID-19: An Italian multicenter experience.Int J Cardiol . 2020.

\section{Hosted file}

Figure 1.pdf available at https://authorea.com/users/390494/articles/504818-prior-use-oftherapeutic-anticoagulation-does-not-protect-against-covid-19-related-clinical-outcomesin-hospitalized-patients-a-propensity-score-matched-cohort-study

\section{Hosted file}

Table 1.pdf available at https://authorea.com/users/390494/articles/504818-prior-use-oftherapeutic-anticoagulation-does-not-protect-against-covid-19-related-clinical-outcomesin-hospitalized-patients-a-propensity-score-matched-cohort-study

\section{Hosted file}

Table 2.pdf available at https://authorea.com/users/390494/articles/504818-prior-use-oftherapeutic-anticoagulation-does-not-protect-against-covid-19-related-clinical-outcomesin-hospitalized-patients-a-propensity-score-matched-cohort-study 